\title{
Atovaquone/Proguanil Resistance in an Imported Malaria Case in Chile
}

\author{
Stella M. Chenet, ${ }^{1,2 *}$ Alan Oyarce, ${ }^{2}$ Jorge Fernandez, ${ }^{2}$ Rafael Tapia-Limonchi, ${ }^{1}$ Thomas Weitzel, ${ }^{3}$ Juan R. Tejedo, ${ }^{1,4,5}$ \\ Venkatachalam Udhayakumar, ${ }^{6}$ María Isabel Jercic, ${ }^{2}$ and Naomi W. Lucchi ${ }^{6}$ \\ ${ }^{1}$ Instituto de Enfermedades Tropicales, Universidad Nacional Toribio Rodríguez de Mendoza (UNTRM), Chachapoyas, Perú; ${ }^{2}$ Instituto de Salud \\ Pública de Chile (ISP), Santiago, Chile; ${ }^{3}$ Facultad de Medicina Clínica Alemana, Universidad del Desarrollo, Santiago, Chile; ${ }^{4}$ Departamento de \\ Biología Molecular e Ingeniería Bioquímica, Universidad Pablo de Olavide (UPO), Seville, Spain; ${ }^{5}$ Diabetes and Associated Metabolic Diseases \\ Networking Biomedical Research Centre (CIBERDEM), Madrid, Spain; ${ }^{6}$ Malaria Branch, Division of Parasitic Diseases and Malaria, Centers for
} Disease Control and Prevention, Atlanta, Georgia

\begin{abstract}
In November 2018, we diagnosed a cluster of falciparum malaria cases in three Chilean travelers returning from Nigeria. Two patients were treated with sequential intravenous artesunate plus oral atovaquone/proguanil (AP) and one with oral AP. The third patient, a 23-year-old man, presented with fever on day 29 after oral AP treatment and was diagnosed with recrudescent falciparum malaria. The patient was then treated with oral mefloquine, followed by clinical recovery and resolution of parasitemia. Analysis of day 0 and follow-up blood samples, collected on days 9, 29, 34, 64, and 83 , revealed that parasitemia had initially decreased but then increased on day 29. Sequencing confirmed Tyr268Cys mutation in the cytochrome $b$ gene, associated with atovaquone resistance, in isolates collected on days 29 and 34 and $P$. falciparum dihydrofolate reductase mutation Asn51lle, associated with proguanil resistance in all successfully sequenced samples. Molecular characterization of imported malaria contributes to clinical management in non-endemic countries, helps ascertain the appropriateness of antimalarial treatment policies, and contributes to the reporting of drug resistance patterns from endemic regions.
\end{abstract}

Chile is one of the South American countries that are free of endemic malaria, without autochthonous cases since April $1945 .^{1}$ Although malaria has been eliminated in Chile, globalization and cultural exchange have contributed to raising awareness of the disease. Moreover, with the recent increase in imported malaria cases and the worldwide spread of drug resistance, the national health reference center in Chile (Instituto de Salud Pública [ISP]) has improved its malaria surveillance, which now includes molecular species identification from all samples and analyses of possible resistance markers in cases of clinical treatment failure. ${ }^{2}$ Malaria is a disease of immediate notification in Chile as part of the Emerging Diseases Surveillance Program of the Ministry of Health.

Currently, artemisinin-based combination therapies are the first-line treatment for Plasmodium falciparum in most endemic regions. ${ }^{3}$ Nevertheless, in Chile, atovaquone/proguanil (AP) is used as an alternative treatment. ${ }^{4}$ Moreover, AP is the most widely used regimen for the prevention of malaria in travelers to endemic countries. ${ }^{5}$ Previous case reports and studies have shown that resistance to atovaquone is associated with point mutations of the $P$. falciparum mitochondrial cytochrome b gene (Pfcytb) (most commonly Tyr268Ser) and resistance to proguanil is associated with point mutations in the $P$. falciparum dihydrofolate reductase gene (Pfdhfr- also relevant for antifolate resistance) such as N51I, C59R, and S108N. ${ }^{6-8} A$ high rate of antifolate resistance is currently found in P. falciparum parasites across major areas of endemicity ${ }^{2,7,9}$; however, the Tyr268Ser mutation in Pfcytb has been uncommonly reported in samples collected before therapy. ${ }^{10,11}$ Interestingly, a report of results from a rodent model suggested that mutations in cytb may not be able to naturally spread within a Plasmodium population because of an associated fitness cost. $^{12}$ However, these mutations can be selected by drug pressure and arise after treatment. Moreover, reported AP treatment failures tend to follow a specific pattern, in which

*Address correspondence to Stella M. Chenet, Instituto de Enfermedades Tropicales, Universidad Nacional Toribio Rodriguez de Mendoza de Amazonas, Chachapoyas, Peru. E-mail: stella.chenet@ untrm.edu.pe some parasites that are originally proguanil-resistant but atovaquone-sensitive develop de novo mutations that confer resistance during the acute infection, leading to late recrudescence after treatment. ${ }^{6,7}$ This does not exclude the possibility of Pfcytb mutations carried by parasite subpopulations, where the mutation cannot be detected because of its low frequency, or the spread of parasites with permissive genetic backgrounds that favor Pfcytb mutation following drug pressure. Investigation of AP treatment failures is important, especially in non-endemic countries that treat imported cases and require information about; here, we describe the first APresistant imported malaria case in Chile.

In November 2018, three members of an Afro-Andean musical group presented at different health centers in Santiago, Chile, with an acute febrile illness accompanied by other symptoms such as myalgia, headache, vomiting, and jaundice. The members of the group had participated for 2 weeks in a music festival in Nigeria, without taking malaria prophylaxis, and returned to Chile on October 27; each was diagnosed with falciparum malaria by thick and thin smears and rapid diagnostic tests. As a notifiable disease, the samples were sent to the ISP, where the diagnosis was confirmed by real-time PCR (qPCR). ${ }^{13}$ Because of the severity of their clinical presentation (renal failure), two of the patients, a 33year-old woman and a 40-year-old man, were treated with sequential intravenous artesunate and oral AP. Both recovered after being in the intensive care unit.

The third patient, a 23-year-old man, reported the first symptoms on November 1 and attended the hospital 5 days later. He received a standard oral treatment schedule with AP (1,000 mg/400 mg once daily for 3 days), with adequate clinical response. However, on day 29 after treatment, he presented with fever, myalgia, and headache and was readmitted with clinical suspicion of falciparum recrudescence. Microscopic examination of a blood smear confirmed $P$. falciparum parasitemia. The patient was treated with oral $250 \mathrm{mg}$ mefloquine tablets (initial three tablets, then two tablets after 8 hours, and then one tablet after 24 hours) and followed up for up to 83 days when an adequate clinical and parasitological response was 


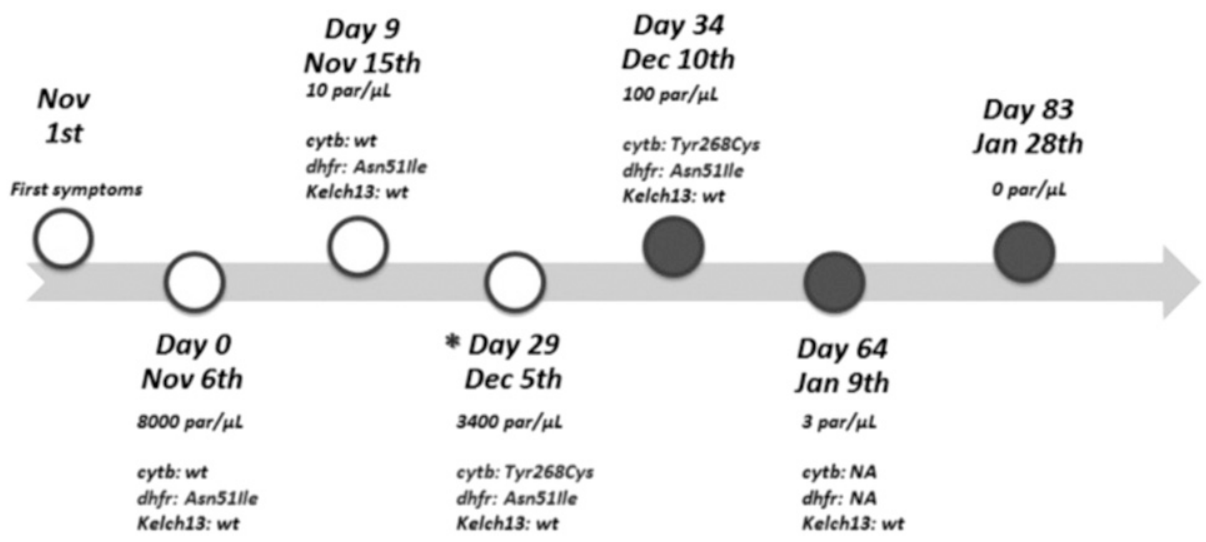

FIGURE 1. Timeline of patient's history since the onset of symptoms and follow-up days after treatment. Days of sample collection during the follow-up after treatment are shown with real-time PCR results expressed in parasites $/ \mu \mathrm{L}$ (par/ $\mu \mathrm{L})$. Full circles represent samples after the change of treatment to mefloquine on day 29. NA = no amplification. ${ }^{*}$ Patient presented with recrudescent malaria.

observed, according to Chilean treatment guidelines. ${ }^{4}$ Available venous blood samples (day 0-the day of initial treatment, days 9, 29, 34, 64, and 83) from this patient were sent to the ISP for diagnosis confirmation and parasite quantification using $\mathrm{qPCR}$ and evaluation of molecular markers of drug resistance. PCR amplification and Sanger sequencing of the Pfcytb (a 650-bp fragment spanning codons 268-278), Pfdhfr (codons 51, 59, 108, and 164), and the PfKelch13 (propeller domain and codon positions 440-600), associated with atovaquone, proguanil, and artemisinin resistance, respectively, were performed for all the seven samples of the third patient, using previously reported methods. ${ }^{7,14}$

Samples from days 0 and 9 showed a reduction in parasite density from 8,000 parasites $/ \mu \mathrm{L}$ to 10 parasites $/ \mu \mathrm{L}$, respectively, according to qPCR results. However, the sample from day 29 showed an increased parasitemia of 3,400/ $\mathrm{L}$, which together with the clinical manifestations confirmed a treatment failure because the patient did not travel outside Santiago after the initial malaria episode. These results are consistent with previous reports which predict that recrudescence in patients with Pfcytb (codon 268)-mutant parasites occurs, on average, on day 29 (95\% Cl: 22, 35) after treatment. ${ }^{15}$ Samples collected after mefloquine treatment showed a parasitemia reduction, with complete parasite clearance on day 83 (Figure 1). On day 64,3 parasites/ $\mu L$ were reported by qPCR but not by microscopy; these results could be explained by DNA persistence after parasite clearance.

Sequencing results of the Pfcytb gene revealed a Tyr268Cys mutation in samples from days 29 to 34 , although results from the earlier samples (days 0 and day 9) showed the wildtype allele. The Pfdhfr mutation Asn51lle was present in all samples before and after the treatment. We neither observed mutations at codons 59 and 108 that are commonly observed in Africa nor the rare 164L mutation. No mutations were observed in the PfKelch13 gene. The results were consistent with the clinical and parasitological disease course, specifically the reduction of the parasite load after AP treatment, representing the decline of Pfcytb wild-type population, but subsequent replication of the population with the Tyr268Cys polymorphism leading to parasitological and clinical recrudescence of the disease. On the other hand, day 0 and day 7 samples from the other two patients, who were adequately treated with no recrudescence, did not present any mutations in Pfcytb or
PfKelch13 but did harbor the Pfdhfr mutation Asn51lle. The presence of the Pfdhfr Asn51lle allele was not surprising, given that this allele is present in Nigeria, where these isolates were acquired. ${ }^{7}$ Our results suggest that Pfcytb mutant parasites should be suspected in a recrudescent infection after AP treatment and a change in treatment should be considered.

This report represents the first reported AP resistance in an imported case of falciparum malaria in Chile. The study highlights the usefulness of collecting accurate information on patient travel history, which is fundamental to diagnose malaria promptly and for provision of effective treatment. In addition, the molecular surveillance of imported malaria in non-endemic regions contributes to the monitoring of emerging drug resistance in endemic countries as it often reflects the situation where the parasites were acquired. The development of drug resistance poses one of the greatest threats to malaria control, and non-endemic countries need to consider this in their national treatment guidelines. Given the fact that recrudescent malaria has been reported after AP treatment, it might be worth for non-endemic countries to develop some guidelines on the recommended treatment of malaria patients returning from Africa. Consideration of alternative treatments such as Coartem or mefloquine should be considered.

This report also highlights the need to advertise and encourage the use of chemoprophylaxis in travelers visiting high-transmission malaria areas. In conclusion, a close followup of antimalarial treatment response is key in non-endemic countries as it allows for the detection of polymorphisms associated with drug resistance, which in turn helps ascertain the appropriateness of antimalarial treatment policies of imported cases and provides information about drug-resistant genotypes entering countries.

Received August 28, 2020. Accepted for publication January 22, 2021.

Published online March 29, 2021.

Acknowledgment: We thank all members of the Parasitology Laboratory and the Molecular Genetics Laboratory of ISP.

Financial support: S. M. C, J. R. T., and R. T.-L. are supported by the National University Toribio Rodríguez de Mendoza (Chachapoyas, Peru) (Grants: Contrato No 09-2019-FONDECYT-BM-INC.INV). 
Disclaimer: The opinions expressed herein are those of the authors and do not necessarily reflect the views of the CDC.

Authors' addresses: Stella M. Chenet, Instituto de Enfermedades Tropicales, Universidad Nacional Toribio Rodriguez de Mendoza de Amazonas, Chachapoyas, Peru, and Sección de Parasitología, Instituto de Salud Publica de Chile, Santiago, Chile, E-mail: schenet@ asu.edu. Alan Oyarce and María Isabel Jercic, Sección de Parasitología, Instituto de Salud Publica de Chile, Santiago, Chile, E-mails: aoyarce@ispch.cl and majercic@ispch.cl. Jorge Fernandez, Subdepartamento de Genética Molecular, Instituto de Salud Pública de Chile (ISP), Santiago, Chile, E-mail: jfernand@ispch.cl. Rafael TapiaLimonchi, Instituto de Enfermedades Tropicales, Universidad Nacional Toribio Rodriguez de Mendoza de Amazonas, Chachapoyas, Peru, E-mail: rafael.tapia@untrm.edu.pe. Thomas Weitzel, Facultad de Medicina Clínica Alemana, Universidad del Desarrollo, Santiago, Chile, E-mail: thomas.weitzel@gmail.com. Juan R. Tejedo, Instituto de Enfermedades Tropicales, Universidad Nacional Toribio Rodriguez de Mendoza de Amazonas, Chachapoyas, Peru, and Departamento de Biología Molecular e Ingeniería Bioquímica, Universidad Pablo de Olavide, Sevilla, Spain, E-mail: juan.tejedo@untrm.edu.pe. Venkatachalam Udhayakumar and Naomi W. Lucchi, Malaria Branch, CDC, Chamblee, GA, E-mails: vxu0@cdc.gov and frd9@cdc.gov.

\section{REFERENCES}

1. Schenone F, Hugo, Andrea Olea N, Antonio Rojas S, Nolberto García D, 2002. Malaria en Chile: 1913-2001. Revista Médica de Chile 130: 1170-1176.

2. Escobar DF, Lucchi NW, Abdallah R, Valenzuela MT, Udhayakumar V, Jercic MI, Chenet SM, 2020. Molecular and epidemiological characterization of imported malaria cases in Chile. Malar J 19: 289.

3. World Health Organization, 2015. Guidelines for the Treatment of Malaria. Geneva, Switzerland: WHO. Available at: https:// www.who.int/malaria/publications/atoz/9789241549127/en/. Accessed May 4, 2020.

4. MINSAL, 2019. Orientaciones técnicas para el diagnóstico y tratamiento de la malaria en Chile Santiago. Available at: https:// diprece.minsal.cl/wrdprss_minsal/wp-content/uploads/2016/
06/Orientaciones-t\%C3\%A9cnicas-para-el-diagn\%C3\%B3sticoy-tratamiento-de-la-Malaria.pdf. Accessed March 3, 2020.

5. World Health Organization, 2016. World Malaria Report. Geneva, Switzerland: WHO. Available at: www.who.int/malaria. Accessed March 3, 2020.

6. Musset L, Le Bras J, Clain J, 2007. Parallel evolution of adaptive mutations in Plasmodium falciparum mitochondrial DNA during atovaquone-proguanil treatment. Mol Biol Evol 24: 1582-1585.

7. Plucinski $M$ et al., 2014. Novel mutation in cytochrome B of Plasmodium falciparum in one of two atovaquone-proguanil treatment failures in travelers returning from same site in Nigeria. Open Forum Infect Dis 1: ofu059.

8. Parzy D, Doerig C, Pradines B, Rico A, Fusai T, Doury C, 1997. Proguanil resistance in Plasmodium falciparum African isolates: assessment by mutation-specific polymerase chain reaction and in vitro susceptibility testing. Am J Trop Med Hyg 57: 646-650.

9. Massamba L et al., 2020. Late clinical failure associated with cytochrome b codon 268 mutation during treatment of falciparum malaria with atovaquone-proguanil in traveller returning from Congo. Malar J 19: 37.

10. Ekala MT et al., 2007. Sequence analysis of Plasmodium falciparum cytochrome b in multiple geographic sites. Malar J 6: 164.

11. Wichmann $O$ et al., 2004. Screening for mutations related to atovaquone/proguanil resistance in treatment failures and other imported isolates of Plasmodium falciparum in Europe. $J$ Infect Dis 190: 1541-1546.

12. Goodman $C D$ et al., 2016. Parasites resistant to the antimalarial atovaquone fail to transmit by mosquitoes. Science 352: 349-353.

13. Rougemont M, Van Saanen M, Sahli R, Hinrikson HP, Bille J, Jaton $\mathrm{K}, 2004$. Detection of four Plasmodium species in blood from humans by 18S RRNA gene subunit-based and speciesspecific real-time PCR assays. J Clin Microbiol 42: 5636-5643.

14. Chenet SM et al., 2016. Independent emergence of the Plasmodium falciparum kelch propeller domain mutant allele C580Y in Guyana. J Infect Dis 213: 1472-1475.

15. Staines HM, Burrow R, Huei-Yi B, Ster IC, Kremsner PG, Krishna $S$, 2018. Clinical implications of Plasmodium resistance to atovaquone/proguanil: a systematic review and meta-analysis. J Antimicrob Chemoth 73: 581-595. 